\title{
ARTICLE Quantitative efficacy of three antipsychotic drugs for schizophrenia based on a real-world study in China
}

\author{
Lei Zhang ${ }^{1}$, Yan-ge $\mathrm{Li}^{1}$, Shen $\mathrm{He}^{1}$, Yan Zhang ${ }^{1}$, Yi-min $\mathrm{Yu}^{1,2}$, Yan $\mathrm{Li}^{1,2}$, Hui Wen ${ }^{1}$, Ying Qiao ${ }^{1}$, Yi-feng Shen ${ }^{1,2}$ and Hua-fang $\mathrm{Li}^{1,2,3}$
}

\begin{abstract}
Atypical antipsychotics exert remarkable long-term efficacy on the personal and social functions of schizophrenic patients. However, quantitative information on the social function of schizophrenic patients treated with atypical antipsychotics is scarce in the current clinical guidelines. In this study, we established pharmacodynamic models to quantify the time-efficacy relationship of three antipsychotic drugs based on the data from a real-world study conducted in China. A total of 373 schizophrenic patients who received antipsychotic monotherapy with olanzapine $(n=144)$, risperidone $(n=160)$, or aripiprazole $(n=69)$ were selected from a three-year prospective, multicenter study. The follow-up times were 13, 26, 52, 78, 104, 130, and 156 weeks after baseline. A time-efficacy model was developed with nonlinear mixed effect method based on changes in Personal and Social Performance (PSP) score compared with the baseline level. Crucial pharmacodynamic parameters, including maximum efficacy and drug onset time, were used to distinguish the efficacy of the three drugs. We quantified the time course of PSP improvement in patients after treatment with these three antipsychotics: olanzapine, risperidone, and aripiprazole reached an $E_{\max }$ value of $80.3 \%, 68.2 \%$, and $23.9 \%$ at weeks $56.7,29.2$, and 36.8 , respectively. General psychotic symptoms, onset frequency, and illness course were identified as significant factors affecting the efficacy of these drugs. The newly constructed models provide an evidence of the benefit of long-term maintenance therapy with atypical antipsychotics in individualized schizophrenia treatment in China.
\end{abstract}

Keywords: schizophrenia; atypical antipsychotics; olanzapine; risperidone; aripiprazole; social functioning; pharmacodynamic models

Acta Pharmacologica Sinica (2019) 40:1611-1620; https://doi.org/10.1038/s41401-019-0285-x

\section{INTRODUCTION}

Schizophrenia is a serious mental illness with a complex set of debilitating and chronic symptoms. At present, second-generation (atypical) antipsychotics (SGAs) are used as first-line treatments for schizophrenia in the clinical setting owing to their higher tolerability than first-generation (typical) antipsychotics (FGAs) and because they are subjectively preferred by patients [1]. The relative effectiveness of atypical antipsychotics compared with that of FGA has been addressed, and studies examining the effectiveness of SGA drugs have also been conducted [2-5].

The advent of SGAs offered a renewed promise of beneficial long-term outcomes, especially in the social functioning of patients, which is defined as the patients' involvement in social interactions and activities. Social functioning of schizophrenic patients has been recognized as a key outcome indicating treatment success [6]; however, the remission of social functioning difficulties would be a more adequate endpoint for evaluating long-term outcome efficacy [7]. Many studies have indicated that continuous antipsychotic treatment, especially as a maintenance treatment, decreases the risk of relapse and improves social function [8, 9]. The CATIE (Clinical Antipsychotic Trials of Intervention Effectiveness) schizophrenia study conducted by the National Institute of Mental Health (NIMH) is the most wellknown comparative study of the effectiveness of antipsychotic drugs $[10,11]$. However, few real-world studies have evaluated the effectiveness of various atypical antipsychotics in China.

In addition, quantitative information on the long-term social functioning of schizophrenic patients treated with SGAs is scarce in current clinical practice $[12,13]$, and the available information does not reflect the differences in therapeutic efficacies between various drugs. Using data from the Study of Long-term Outcomes for Schizophrenia by Atypical Antipsychotic Treatment in China (SALT-C) study, which is a multicenter, real-world clinical study, we examined the differences in efficacy between three antipsychotics (olanzapine, risperidone, and aripiprazole) to provide a guide for clinicians when choosing an antipsychotic for the individualized treatment of schizophrenia. In this study, we established pharmacodynamic models to quantify the time-efficacy relationship of each antipsychotic drug, identify the relevant influencing factors affecting antipsychotic efficacy, and directly reflect the therapeutic efficacy of each drug. These models will ultimately provide quantitative information to realize individualized schizophrenia treatment in China.

\section{MATERIALS AND METHODS}

Study design

The SALT-C study evaluated the effectiveness and safety of antipsychotic drugs in real-world settings and populations; thus, the

\footnotetext{
${ }^{1}$ Department of Psychiatry, Shanghai Mental Health Center, Shanghai Jiao Tong University School of Medicine, Shanghai 200030, China; ${ }^{2}$ Clinical Research Center, Shanghai Jiao Tong University School of Medicine, Shanghai 200030, China and ${ }^{3}$ Shanghai Key Laboratory of Psychotic Disorders, Shanghai 200030, China Correspondence: Hua-fang Li (Ihlh_5@163.com)
} 
study results can be applied in the routine clinical setting in China. The SALT-C study was registered at https://www.clinicaltrials.gov (identifier: NCT02640911) and produced a large data set of real-world schizophrenia patients in China recruited in an open-label 3-year follow-up clinical trial of widely used atypical antipsychotics. In this prospective, randomized, flexible dose, open-label study conducted by Shanghai Mental Health Center, data were also obtained from the Sixth Hospital of Peking University, Beijing Anding Hospital, Guangzhou Psychiatric Hospital, West China Hospital of Sichuan University, Second Xiangya Hospital, First Affiliated Hospital of Kunming Medical College and Shanghai Luwan Mental Health Center between July 2011 and December 2014. Schizophrenic patients treated with atypical antipsychotics were followed for 3 years. Demographic data, medication history, drug dosage, illness course, and efficacy assessments, including the Positive and Negative Syndrome Scale (PANSS) [14] and the Personal and Social Performance scale (PSP) [15], were collected using a standardized data collection form. All the centers followed the same research protocol, and all psychiatrists received training pertaining to these scales. As a real-world study, the protocol did not limit the dosage of drugs, and we only recorded the actual drug usage in clinical practice. We collected the blood drug concentration from the patients to monitor compliance. The medical history provided by guardians was also taken as important evidence to determine medication compliance.

\section{Participants}

Eligible patients were over 16 years of age; diagnosed with schizophrenia based on the Diagnostic and Statistical Manual of Mental Disorders, Fourth Edition (DSM-IV) or the International Classification of Diseases 10 (ICD 10) by two certificated psychiatrists; and able to take atypical antipsychotic medications. Patients with childbearing potential were required to have a negative pregnancy test at the time of screening. The exclusion criteria included substance dependency, dementia, mental retardation, and Axis I or II significant physical illness. The SALT$C$ study was approved by the Institutional Review Board of the Shanghai Mental Health Center, Shanghai Jiao Tong University School of Medicine. Written informed consent was obtained from all participants and the patients' guardians.

In this study, patients who received olanzapine, risperidone or aripiprazole monotherapy at least 13 weeks after the baseline visit in the SALT-C database were included in the model foundation. An average medication compliance of $>90 \%$ in 1 month was assumed to be good compliance.

\section{Procedures and measures}

After signing written informed consent forms, the potential subjects went through the screening process. Each eligible subject was assessed at 13, 26, 52, 78, 104, 130, and 156 weeks after the start of the study. Assessments included clinical and functional measures. The measures of clinical evolution, as scored by the PANSS, and social functioning, as scored by the PSP, were used in our study. The primary efficacy criterion was the PSP score, which was recently developed to specifically assess social functioning in schizophrenic patients [16]. The ratings were based on the assessment of four objective indicators: (a) socially useful activities, (b) personal and social relationships, (c) self-care, and (d) disturbing and aggressive behaviors. The Chinese version of the PSP rating had good reliability, validity, and sensitivity [17]. Higher PSP scores indicate better functioning. After passing a test on the use of the scales and obtaining high internal concordance (an internal concordance of 0.70 was the minimum required), the psychiatrists were qualified as scorers.

Model establishment

We found that the distribution of the improved percentage of the PSP score from the baseline varied with time and at some time points reached a plateau. Thus, the classic pharmacodynamic $\left(E_{\max }\right)$ model [18] was used as the base model for our data (Formula 1).

The parameters in Formula 1 are $E_{\max }, \mathrm{ET}_{50}$, and $\gamma$. $E_{\max }$ is the theoretical maximal drug effect; $\mathrm{ET}_{50}$ is the time required to reach half of the maximal efficacy, which also represents the rate of drug onset; and $\gamma$ is a shape parameter. If the estimate of $\gamma$ was near 1 , the $\gamma$ value was fixed at 1 to simplify the model. $E_{\text {Typical }}$ is the typical drug efficacy, and time is the time of the observation point. The nonlinear mixed effects model was used to fit the function.

$E_{\text {Typical }}=\frac{E_{\max } \times \text { Time }^{\gamma}}{\mathrm{ET}_{50}^{\gamma}+\text { Time }^{\gamma}}$

Owing to the influence of variations, the values of observed efficacy fluctuate around the values of typical efficacy. Therefore, the measured efficacy values in each investigation consisted of typical efficacy values, interindividual variability, and the residual error (RSE). The interindividual variability of the parameters was assigned a logarithmic normal distribution across the population, and the distribution of pharmacodynamic parameters was defined in Formula 2.

$P_{\mathrm{i}}=P_{\text {pop }} \times \mathrm{e}^{\eta_{\mathrm{i}}}$

$P_{\mathrm{i}}$ is the individual pharmacodynamic parameter, whereas $P_{\text {pop }}$ is the population typical value of the corresponding pharmacodynamic parameter; $\eta_{\mathrm{i}}$ is assumed to be normally distributed, with a mean of 0 and variance of $\omega^{2}$.

The RSE was tested by the additive (Formula 3), proportional (Formula 4), and proportional plus additive (Formula 5) models. The minimum value of the nonlinear mixed effect method (NONMEM) objective function value (OFV) was used as a statistic to choose the RSE model.

$E_{\mathrm{i}, \mathrm{j}}=E_{\text {Typical }, \mathrm{i}, \mathrm{j}}+\varepsilon_{\mathrm{i}, \mathrm{j}, 1}$

$E_{\mathrm{i}, \mathrm{j}}=E_{\text {Typical }, \mathrm{i}, \mathrm{j}} \times\left(1+\varepsilon_{\mathrm{i}, \mathrm{j}, 2}\right)$

$E_{\mathrm{i}, \mathrm{j}}=E_{\text {Typical, } \mathrm{i}, \mathrm{j}} \times\left(1+\varepsilon_{\mathrm{i}, \mathrm{j}, 2}\right)+\varepsilon_{\mathrm{i}, \mathrm{j}, 1}$

where $E_{\mathrm{i}, \mathrm{j}}$ is the efficacy value at the observation time point $\mathrm{j}$ of the individual $\mathrm{i}$. The $\varepsilon_{\mathrm{i}, \mathrm{j}, 1}$ and $\varepsilon_{\mathrm{i}, \mathrm{j}, 2}$ variables are the additive and proportional RSEs in individual $i$ at time point $j$, respectively; $\varepsilon_{i, j, 1}$ and $\varepsilon_{i, j, 2}$ are assumed to be generally distributed with a mean of 0 and variance of $\sigma_{1}{ }^{2}$ and $\sigma_{2}{ }^{2}$, respectively.

Model development and validation

Once the aforementioned basic model was established, potential factors that might affect the model parameters were explored. The factors tested in the study were age, sex, dosage, age at initial onset, illness duration, illness course, schizophrenic episodes, onset frequency, family history, and PANSS score at the baseline. In the process of covariate model building, categorical covariates were modeled according to Formula 6, whereas the continuous covariates were tested by Formula 7 or Formula 8.

$$
\begin{aligned}
& P_{\mathrm{i}}=P_{\text {pop }}+\mathrm{COV} \times \theta_{\mathrm{COV}} \\
& P_{\mathrm{i}}=P_{\text {pop }}+\left(\mathrm{COV}-\mathrm{COV}_{\text {median }}\right) \times \theta_{\mathrm{COV}} \\
& P_{\mathrm{i}}=P_{\text {pop }} \times\left(\mathrm{COV} \div \mathrm{COV}_{\text {median }}\right)^{\theta_{\mathrm{cov}}}
\end{aligned}
$$

In formulas $6-8, P_{\mathrm{i}}$ is the pharmacodynamic parameter for a patient with a covariate value of COV. COV $_{\text {median }}$ is the median value of the covariable in the population. $P_{\text {pop }}$ is the typical value of the parameter when the categorical covariates are equal to 0 or 
continuous covariates are equal to $\operatorname{COV}_{\text {median. }} \theta_{\text {cov }}$ is a correction coefficient of the covariate of the model parameter.

A difference in the OFV of $3.84(P=0.05, \mathrm{df}=1)$ and $6.63(P=$ $0.01, \mathrm{df}=1$ ) was considered statistically significant in the covariate model-building process. All factors as covariates were analyzed in a stepwise manner with a forward selection step $(P=0.05)$ and then a stricter backward elimination step $(P=0.01)$ [19].

Diagnostic plots were assessed to confirm the model performance. The Monte Carlo method was performed 1000 times to generate the $90 \%$ confidence intervals $(\mathrm{Cl})$ of the effects of each medicine. Then, the interval was compared with the observed values to assess the accuracy of the model. Validation of the model was also performed by the bootstrap method [20].

\section{Software and statistical methods}

Statistical analysis was conducted with SPSS (version 24.0, IBM Corp, Armonk, NY, USA). The demographic and clinical characteristics of all the study samples are described with descriptive statistics. Group comparisons were performed with ANOVA or the Kruskal-Wallis $\mathrm{H}$ test for continuous variables and the Fisher exact test for categorical variables.

The model estimation and simulation were performed with NONMEM 7.4 (Level 1.0, ICON Development Solutions, USA). Diagnostic graphics and visual predictive checks were conducted with $\mathrm{R}$ software (version 3.0.1, The R Foundation of Statistical Computing, Austria). A value of $P \leq 0.05$ indicated a statistically significant difference.

\section{RESULTS}

Study characteristics

A total of 373 schizophrenic participants were included in our analysis, of which 144 were on olanzapine, 160 were on risperidone and 69 were on aripiprazole. The characteristics of the patients are summarized in Table 1, and the sample size of each center is presented in Supplementary Table S1. The mean age was 38.0 years, and the percentage of male participants was $53.1 \%$. The PSP score at the baseline was $53.1 \pm 15.6$. We found that age, occupation, illness duration, course, family history, PANSS, and PSP scores at the baseline were significantly different $(P<0.05)$ among the three treatment drug groups.

\section{Model establishment}

In our study, the $E_{\max }$ model described an increased percentage of the PSP score and could describe the time-effect of the three atypical antipsychotic medications. The RSE was best described by the additive error model. The estimated values of $\gamma$ for olanzapine, risperidone and aripiprazole were $1.03,0.94$, and 1.11 , respectively, which are very close to 1 ; thus, we fixed the $\gamma$ values to 1 in the final model. The parameter estimation for the final model is presented in Table 2. The values of RSE for the pharmacodynamic parameters of olanzapine and risperidone ranged from $6.7 \%$ to $20.7 \%$, which were considered acceptable. However, the RSE for the pharmacodynamic parameters of aripiprazole was $>30 \%$, which was owing to the small sample size and few observation time points. After removing the outliers (above the mean \pm 3 standard deviations), the RSE for the pharmacodynamic parameters of aripiprazole was $<30 \%$, and detailed information about the outliers is presented in Supplementary Table S2. Therefore, the estimated values of the pharmacodynamic parameters after removing the outliers were compared with those of the other two drugs.

The typical $E_{\max }$ values of olanzapine, risperidone, and aripiprazole were $80.3 \%, 68.2 \%$, and $23.9 \%$, respectively. The time to achieve half of the maximal effect $\left(E_{50}\right)$ for olanzapine, risperidone, and aripiprazole was 56.7, 29.2, and 36.8 weeks, respectively.
Moreover, we observed that the number of schizophrenic attacks and general psychopathology had a significant effect on the $\mathrm{ET}_{50}$ of olanzapine (Formula 9).

$$
\begin{array}{r}
\mathrm{ET}_{50-\text { olanzapine }}=56.7 \times(\text { General psychopathology } \div 29)^{2.46} \\
\times(\text { Oneset frequency } \div 2)^{1.32}
\end{array}
$$

Formula (9) shows $\mathrm{ET}_{50}$ values at 22.7 weeks for the general psychopathology score of 29 in first-episode patients and 56.7 weeks in first recurrence patients. For patients who had a relapse, the time to reach their half maximal social function increased exponentially. Meanwhile, in first recurrence patients, the $\mathrm{ET}_{50}$ values were 56.7 weeks for a general psychopathology score of 29 and 311.9 weeks for a general psychopathology score of 58. All of these indicated that the number of relapses and the general psychopathology score were closely related to the $\mathrm{ET}_{50}$. For example, when the general psychopathology score is 16 , and the onset frequency is 1 , the $\mathrm{ET}_{50}$ value of olanzapine is 5.3 weeks. When the general psychopathology score is 40 , and the onset frequency is 4, the $\mathrm{ET}_{50}$ value of olanzapine is 312.5 weeks.

Episodes of schizophrenia and the total PANSS total score had significant effects on the $E_{\max }$ and the illness course (months) had a significant effect on the $\mathrm{ET}_{50}$ of risperidone (Formulas 10 and 11).

$$
E_{\text {max-risperidone }}=(68.2-0.748(\text { PANSS }-58))-33.3 \times \text { Episode }
$$

$\mathrm{ET}_{50-\text { risperidone }}=29.2+($ Course -7.55$) \times 0.524$

Formula (10) showed $E_{\max }$ values of $68.2 \%$ for a PANSS score of 58 in first-episode patients and $35.0 \%$ in relapsing patients undergoing risperidone treatment. For example, when the PANSS score was 60 , and the episode was 1, the $E_{\max }$ value of risperidone was $33.3 \%$. When the PANSS score was 90 , and the episode was 2 , the $E_{\max }$ value of risperidone was $22.3 \%$.

Formula (11) showed $\mathrm{ET}_{50}$ values of $26.8,28.3$ and 31.5 weeks for illness courses of 3,6 , and 12 months, respectively.

\section{Model evaluation}

Figure 1 depicts the goodness-of-fit of the final models. The observed (OBS) effect was in good accordance with the population-predicted effect data (PRED) effects and the individual predicted data (IPRED) effects. The WRES magnitude was located within \pm 4 from the center and distributed randomly around the overall range of the PRED. The visual predictive check with Monte Carlo simulation showed that the mean PSP score increase (\%) values for most actual values were distributed within the predicted $5 \%$ and $95 \%$ boundaries for all three atypical antipsychotics (Fig. 2). Bootstrap sampling also indicated the stability of the model by showing the similarities between the medians and the estimated values of the final pharmacodynamics parameters (Tables 2,3). The $95 \% \mathrm{Cl}$ of $\theta$ did not contain 0 , suggesting that the factors were the significant factors influencing the parameters $\mathrm{ET}_{50}$ and $E_{\text {max }}$. All of these evaluation results indicated good predictability of the final models.

\section{Typical drug efficacy values}

According to the final model parameters, we examined the efficacy values of the three antipsychotics at 26, 52, 78, 104, 130, and 156 weeks and simulated the typical efficacy-time curves (Fig. 3a). The results showed that olanzapine had the best efficacy, with an actual maximal effect value of $80.3 \%$.

The effect of onset frequency on long-term functioning may be particularly pronounced in patients treated with olanzapine or risperidone. A significant correlation between the number of relapses and the general psychopathology score was observed for patients treated with olanzapine. A three-dimensional curved surface can predict the typical effect of olanzapine with different 
Table 1. Characteristics of three groups

\begin{tabular}{|c|c|c|c|c|c|}
\hline & $\begin{array}{l}\text { Olanzapine } \\
n=144\end{array}$ & $\begin{array}{l}\text { Risperidone } \\
n=160\end{array}$ & $\begin{array}{l}\text { Aripiprazole } \\
n=69\end{array}$ & $\begin{array}{l}\text { Overall } \\
n=373\end{array}$ & $P$ value \\
\hline Age (year) & $36.4 \pm 14.9$ & $41.0 \pm 13.5$ & $32.1 \pm 12.3$ & $38.0 \pm 14.2$ & 0.000 \\
\hline Male $n(\%)$ & $81(56.3)$ & $74(46.3)$ & $43(62.3)$ & $198(53.1)$ & 0.051 \\
\hline BMI & $22.5 \pm 4.0$ & $22.9 \pm 4.0$ & $23.5 \pm 4.3$ & $22.9 \pm 3.9$ & 0.281 \\
\hline Weight $(\mathrm{kg})$ & $61.8 \pm 11.0$ & $64.3 \pm 11.7$ & $63.6 \pm 13.0$ & $63.1 \pm 11.7$ & 0.224 \\
\hline Single $n(\%)$ & $82(56.9)$ & $97(60.6)$ & $36(52.2)$ & $215(57.6)$ & 0.417 \\
\hline Married $n(\%)$ & $55(38.2)$ & $59(36.9)$ & $32(46.4)$ & $146(39.1)$ & - \\
\hline Divorced or widowed $n(\%)$ & $7(4.9)$ & $4(2.5)$ & $1(1.4)$ & $12(3.2)$ & - \\
\hline \multicolumn{6}{|l|}{ Occupation } \\
\hline Employed $n(\%)$ & 56 (38.9) & $59(36)$ & $40(58.0)$ & $155(41.6)$ & 0.009 \\
\hline \multicolumn{6}{|l|}{ Episode } \\
\hline First episode $n(\%)$ & $49(34.0)$ & $35(21.9)$ & $20(29.0)$ & $104(27.9)$ & 0.060 \\
\hline Onset frequency & $3.1 \pm 3.1$ & $3.4 \pm 2.7$ & $2.6 \pm 2.0$ & $3.1 \pm 2.7$ & 0.109 \\
\hline \multicolumn{6}{|l|}{ Family history } \\
\hline Positive (\%) & $43(29.1)$ & $32(19.0)$ & $9(12.2)$ & $84(22.5)$ & 0.021 \\
\hline \multicolumn{6}{|l|}{ PANSS score at baseline } \\
\hline Total score & $69.5 \pm 20.0$ & $72.0 \pm 22.6$ & $64.2 \pm 20.2$ & $69.6 \pm 21.3$ & 0.038 \\
\hline Positive score & $16.8 \pm 6.8$ & $17.4 \pm 8.1$ & $14.6 \pm 6.2$ & $16.7 \pm 7.4$ & 0.027 \\
\hline Negative score & $18.1 \pm 6.4$ & $19.8 \pm 7.4$ & $17.0 \pm 7.5$ & $18.6 \pm 7.1$ & 0.013 \\
\hline General psychopathology & $34.5 \pm 11.1$ & $34.8 \pm 11.0$ & $32.6 \pm 10.3$ & $34.3 \pm 10.9$ & 0.356 \\
\hline
\end{tabular}

Table 2. Parameter estimation of the three drugs

\begin{tabular}{|c|c|c|c|c|}
\hline \multicolumn{5}{|c|}{ Pharmacodynamics parameters } \\
\hline$E_{\max } \%$ & $80.3(6.7)$ & $68.2(11.4)$ & $44.2(38.9)$ & 23.9(17.6) \\
\hline $\mathrm{ET}_{50}$, week & $56.7(16.4)$ & $29.2(20.7)$ & $49.7(57.9)$ & $36.8(29.8)$ \\
\hline \multicolumn{5}{|l|}{ Covariate parameters } \\
\hline$\theta 1$ on $E_{\max }$ & NA & $-33.3(18.2)$ & NA & NA \\
\hline$\theta 4$ on $\mathrm{ET}_{50}$ & NA & $0.524(62.2)$ & NA & NA \\
\hline$\theta 5$ on $\mathrm{ET}_{50}$ & $2.46(13.7)$ & NA & NA & NA \\
\hline \multicolumn{5}{|l|}{ Variability parameters } \\
\hline$\eta\left(E_{\max }\right), \%$ & $22.4(17.9)$ & $28.0(28.4)$ & $58.5(11.7)$ & $56.9(13.7)$ \\
\hline$\eta\left(\mathrm{ET}_{50}\right), \%$ & $119.6(10.2)$ & $110.5(12.5)$ & $69.9(21.3)$ & - \\
\hline Shrinkage $\_\eta\left(\mathrm{ET}_{50}\right), \%$ & 34.1 & 40.2 & 53.6 & - \\
\hline Shrinkage _e, \% & 9.7 & 8.9 & 12.6 & 11.5 \\
\hline
\end{tabular}



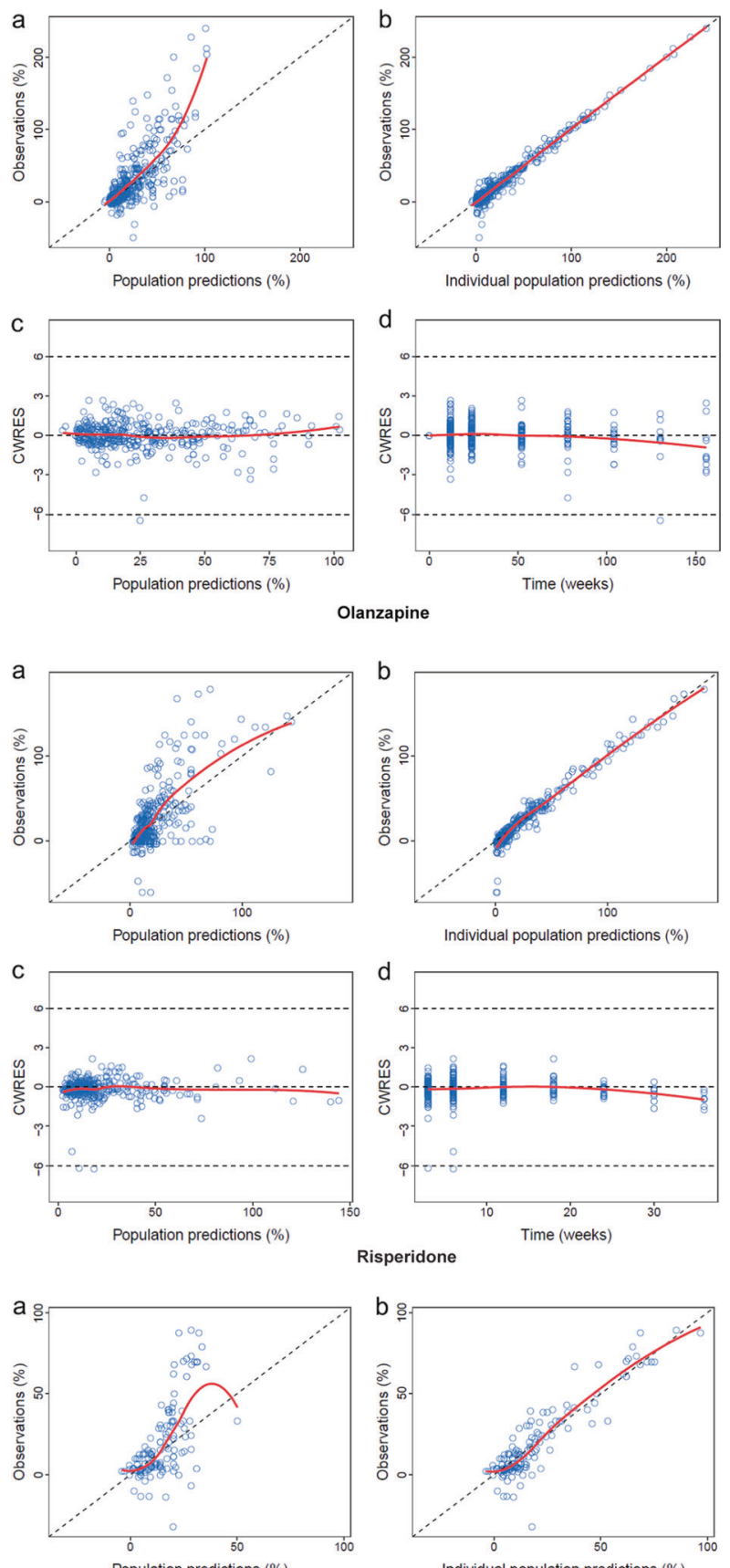

Population predictions (\%)

Individual population predictions $(\%)$
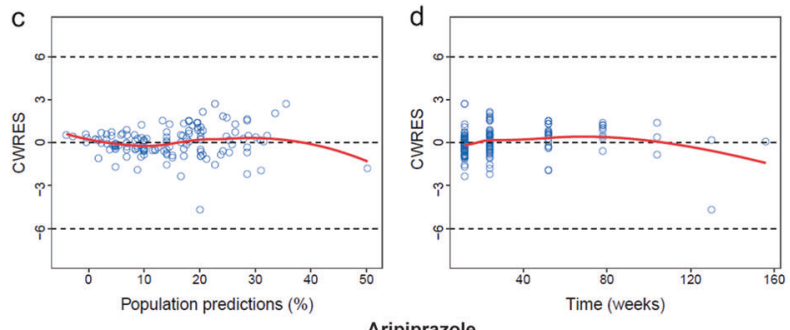

Aripiprazole

onset frequencies for the general psychopathology score of 29 (Fig. 4a). The course-time-effect model of risperidone shows a better effect in first-episode patients (Fig. 4b) than in relapsing patients (Fig. 4c). Moreover, no factor was significantly correlated with $E_{\max }$ or $\mathrm{ET}_{50}$ in the aripiprazole group.
Fig. 1 The goodness-of-fit plots for the final models. a Scatter plot of population-predicted effect data (PRED) vs. observed effect data (OBS). b Scatter plot individual predicted effect data (IPRED) vs. OBS. c Plot of conditional weighted residuals (CWRES) vs. PRED. d Plot of CWRES vs. the Time point. The black and red lines in a and b represent identity and regression lines, respectively, whereas in c and d, the black lines are the position where conditional weighted residual equal 0 and the red lines are the regression lines. The goodness-of-fit plots for the aripiprazole are plots after removing the outliers

Treatment discontinuation rate

The duration for which patients continue to use a drug is considered a good measure of drug effectiveness [21]. Therefore, we conducted a statistical analysis of the treatment discontinuation rate (TDR) for the three drugs at different time points (Table 4). The results showed that risperidone had the highest allcause TDR. TDR in schizophrenia patients treated with olanzapine was substantially lower than that in patients receiving aripiprazole before 24 months but was higher than that in patients taking aripiprazole after 24 months (Fig. 3b).

\section{DISCUSSION}

In this prospective study of real-world schizophrenic patients treated with long-term antipsychotic monotherapy, maintenance treatment generally improved the social functioning of patients. We chose these three antipsychotics because of their widespread global use and their different receptor profiles, as well as because their efficacy has not been quantitatively assessed and compared. These three drugs are used for schizophrenia treatment. As traditional clinical efficacy trials usually rely on carefully planned treatment-optimizing protocols with strict inclusion and exclusion criteria [22, 23], meta-analyses or conventional group comparison analyses cannot clearly illustrate the different characteristics of antipsychotic efficacy.

We quantitatively analyzed the therapeutic effects of three antipsychotics on the social functioning of real-world schizophrenic patients using a pharmacodynamic model. We found that the schizophrenic episode and total PANSS total score had a significant effect on the $E_{\max }$ of risperidone. When the firstepisode patients had a PANSS score of 58 , the $E_{\max }$ values of olanzapine, risperidone and aripiprazole were $80.3 \%, 68.2 \%$, and $23.9 \%$, respectively. The typical efficacy-time curves showed that aripiprazole possessed the lowest efficacy, with olanzapine showing much greater therapeutic efficacy than aripiprazole. This result agrees with the findings of an open randomized clinical trial conducted in 14 countries, which suggested that the effectiveness of olanzapine is superior to that of other antipsychotic drugs [5]. A meta-analysis also indicated that olanzapine and risperidone are significantly superior to other drugs in terms of overall efficacy [24]. Our model was thus successful in quantitatively analyzing the difference in effects among the three drugs.

The number of schizophrenic episodes and general psychopathology significantly affected the $\mathrm{ET}_{50}$ of olanzapine, whereas the illness course (months) significantly affected the $\mathrm{ET}_{50}$ of risperidone. The $\mathrm{ET}_{50}$ values of olanzapine were 22.7 weeks in firstepisode patients with a general psychopathology score of 29, 56.7 weeks in first recurrence patients, and 311.9 weeks in first recurrence patients with a general psychopathology score of 58 . The $\mathrm{ET}_{50}$ values of risperidone were $26.8,28.3$, and 31.5 weeks in patients with an illness course of 3, 6, and 12 months, respectively.

In the final model, the RSE of $E_{\max }$ and $\mathrm{ET}_{50}$ for aripiprazole was $>30 \%$. Possible explanations are the relatively large individual variation, small sample size and insufficient observation points. The outliers, which were defined as above the mean \pm 3 standard deviations, had an impact on the model. As the outliers were 

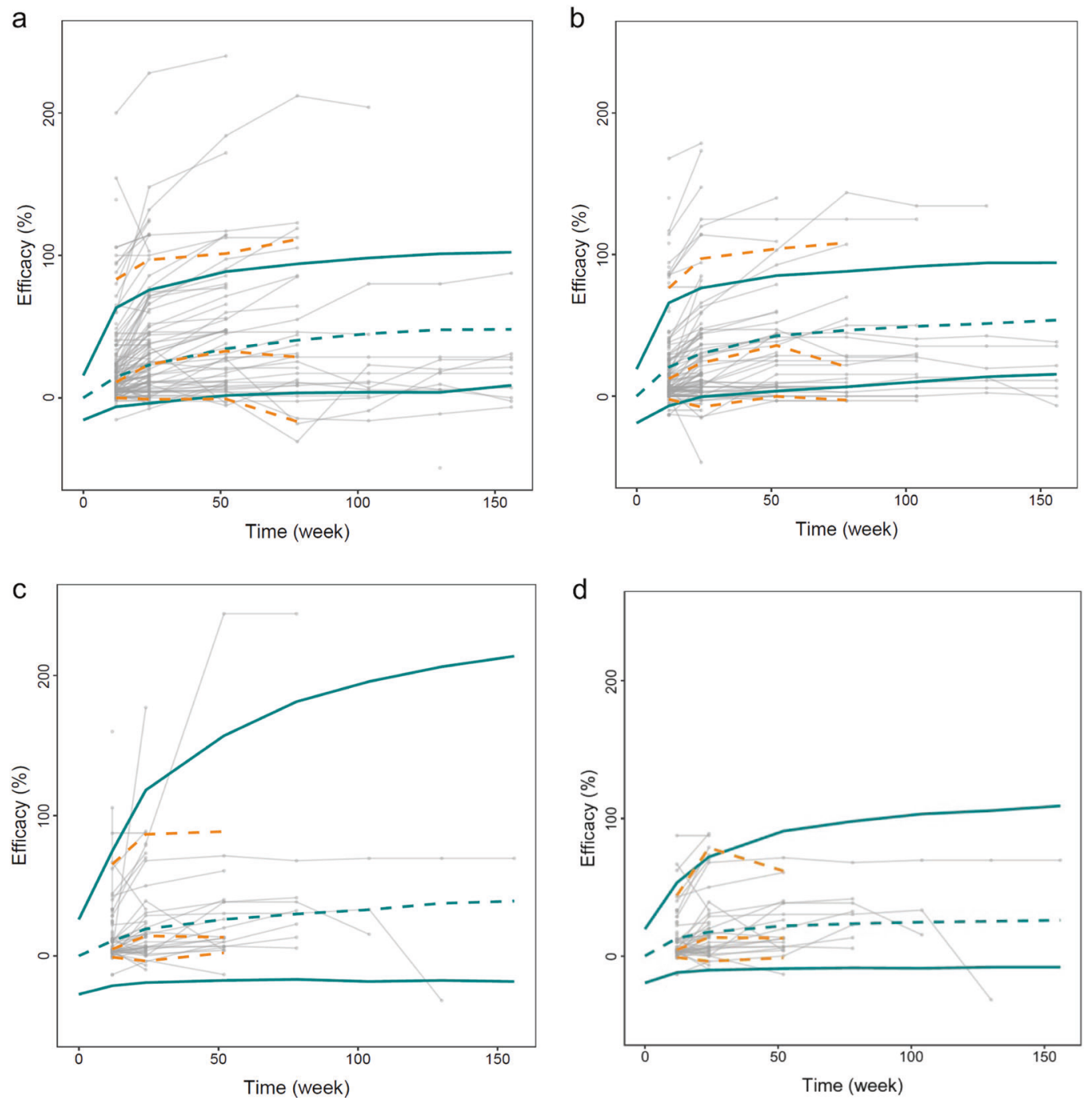

Fig. 2 The visual predictive check for the final model. The dots are the mean values of PSP score change (\%) from each time visit. The population-predicted profile (50th percentile) estimated from 1000 Monte Carlo simulations is shown by the green dashed line, and the $90 \%$ prediction intervals are encompassed by the green solid lines in each plot. The yellow dashed lines are the 5th, 50th, and 95th percentiles of the observed data. a olanzapine; $\mathbf{b}$ risperidone; $\mathbf{c}$ aripiprazole (original data set); $\mathbf{d}$ aripiprazole (outliers remove)

fewer than $10 \%$ of the total, we removed them. After removing the outliers, the precision of estimation was improved. Therefore, the large individual variation was the reason for the poor precision of the original estimates.

Previous studies included many factors, such as sociodemographic factors, treatment adherence, illness duration, and cognitive function, to screen for potential outcome predictors [25-27]. It is unclear whether the collinearity effect between the factors was excluded. Our study used quantitative pharmacological methods to show that factors such as schizophrenic episodes, total PANSS score, the number of schizophrenic attacks and general psychopathology significantly affect the efficacy of olanzapine and risperidone, excluding the collinearity effect. There were some differences in results between our study and previous studies because of the use of different baseline characteristics and because we focused on real-world data.

The effect of onset frequency on the social functioning of schizophrenic patients has been studied. A study showed that after acute treatment, almost half of first-episode schizophrenia patients achieved symptom remission and had adequate social functioning, showing substantial improvement compared to relapsing patients [28]. Every relapse of schizophrenic psychosis is accompanied by an immense burden on the patients themselves, their families and society at large. In our study, we observed that for each increase in the number of schizophrenic attacks, the social function change rate decreased by $\sim 10 \%$ in the olanzapine group, and the first episode was two times more influential than in patients with recurrence in the risperidone group. Our results showed an association between high relapse frequency and poor long-term outcomes, which was consistent with the results of previous studies. A possible explanation is that repeated relapse is always associated with poor drug compliance. Thus, the stability of neurotransmitters such as dopamine and hydroxytryptamine is influenced by poor drug compliance, which is consistent with the hypothesis of schizophrenia pathogenesis [29]. Aripiprazole, unlike olanzapine and risperidone, is a partial agonist of $\mathrm{D}_{2}$ and $5-\mathrm{HT}_{1 \mathrm{~A}}$ receptors, which is different from olanzapine and risperidone. Moreover, neuroimaging studies have shown structural and functional changes in brain regions with extended periods of relapse [30]. Therefore, clinicians should emphasize the importance of compliance and the negative effect of relapse on social functioning in schizophrenia patients. In addition, a shorter duration of psychosis before study entry predicts both full 
Table 3. Bootstrap sampling of the three drugs

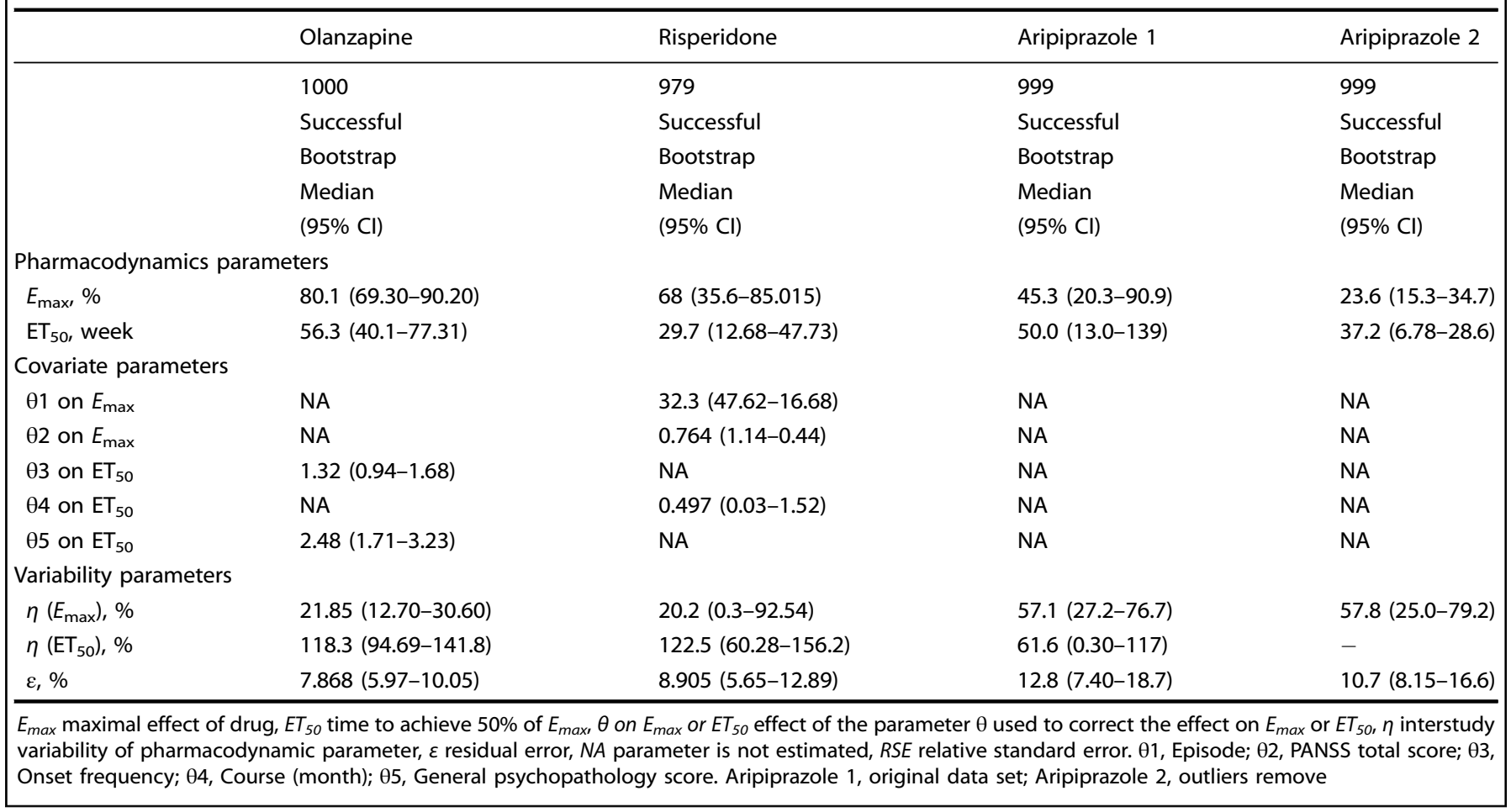
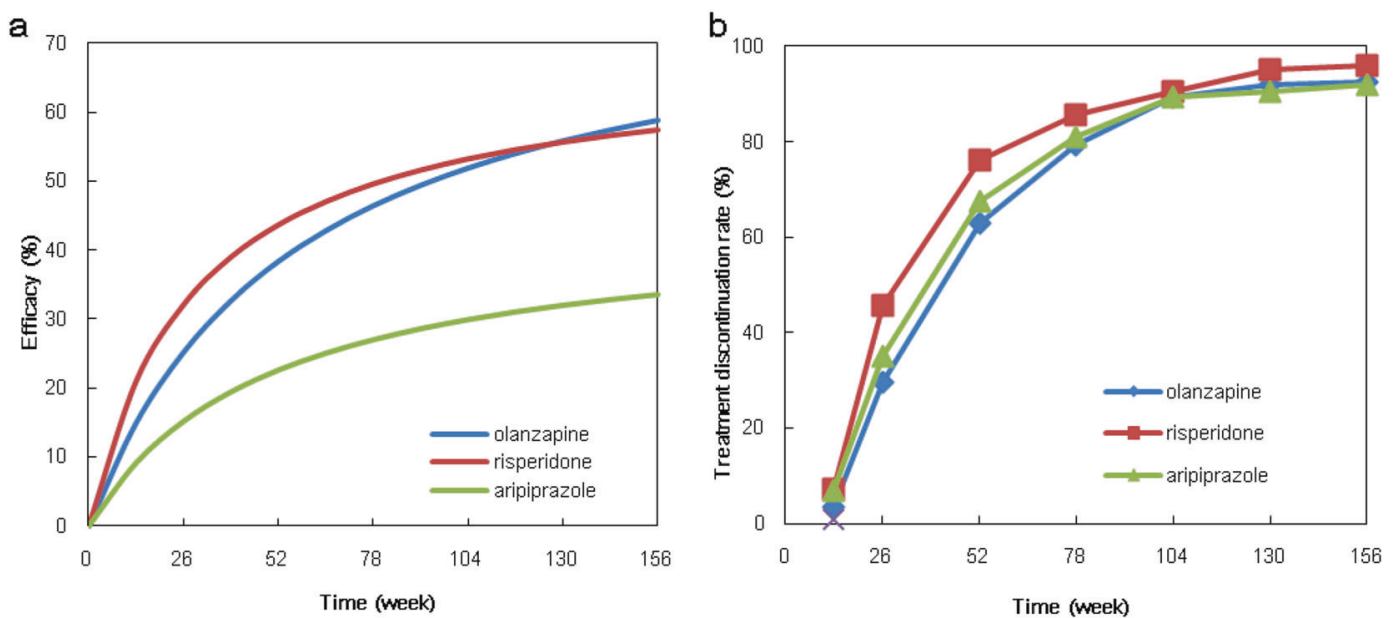

Fig. 3 a The predicted typical PSP score elevation rate of each group. $\mathbf{b}$ The treatment discontinuation rate of the three drugs

recovery and symptom remission [28]. Emerging evidence emphasizes the association between early detection and the long-term outcome [31]. Our study supported the importance of patient-specific factors, including illness severity at the time of diagnosis, onset frequency, and illness course as determinants of long-term antipsychotic response.

The predicted $E_{\max }$ value was not significantly correlated with the dose of each drug. The OFV did not significantly decrease when the dose factor was included in the model parameters, indicating that there was no significant correlation between drug dosage and efficacy, although the dose varies in clinical practice (Fig. 5). We observed that the drug dose decreased rapidly after 2 years. Dose reduction of atypical antipsychotics is more recommended by clinicians than acute doses in the schizophrenia maintenance phase $[9,32]$. Some experts also proposed that maintenance doses should be slowly reduced; moreover, studies have recommended maintaining the dose of an antipsychotic drug for at least 18 months [13]. Finally, dose was not included in our model.

We analyzed the TDR of the three drugs at different time points because the TDR is considered an composite representative of drug efficacy, safety, and tolerability by both the clinicians and the patients $[10,21,33]$. A previous study indicated that olanzapine is the most effective drug for chronic schizophrenia [10]. In our study, of the three long-term SGAs, risperidone showed the highest dropout rate within one year, indicating low patient adherence. The TDR in patients receiving olanzapine was lower than that in patients receiving aripiprazole before 24 months but was higher than that in patients receiving aripiprazole after 24 months. The reason for the superiority of aripiprazole as a longterm continuous treatment may be related to its unique pharmacological profile [34]. Aripiprazole is well tolerated with 
a

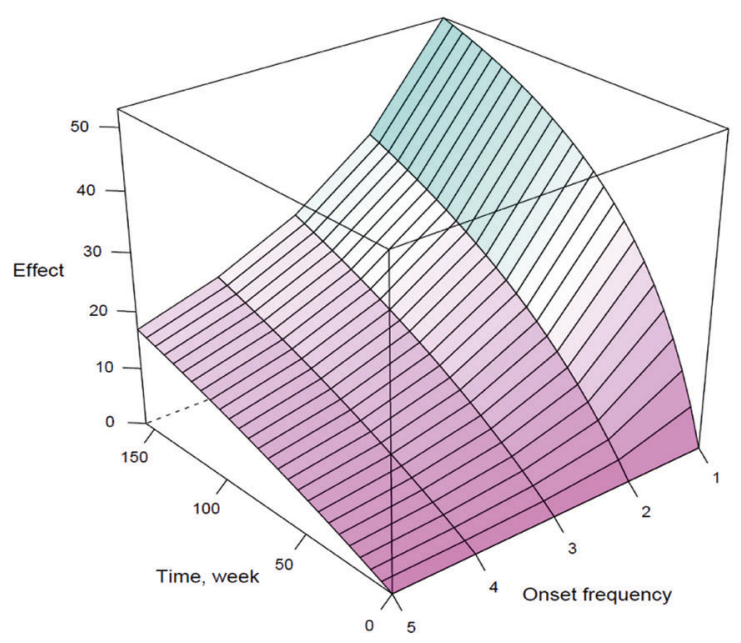

b

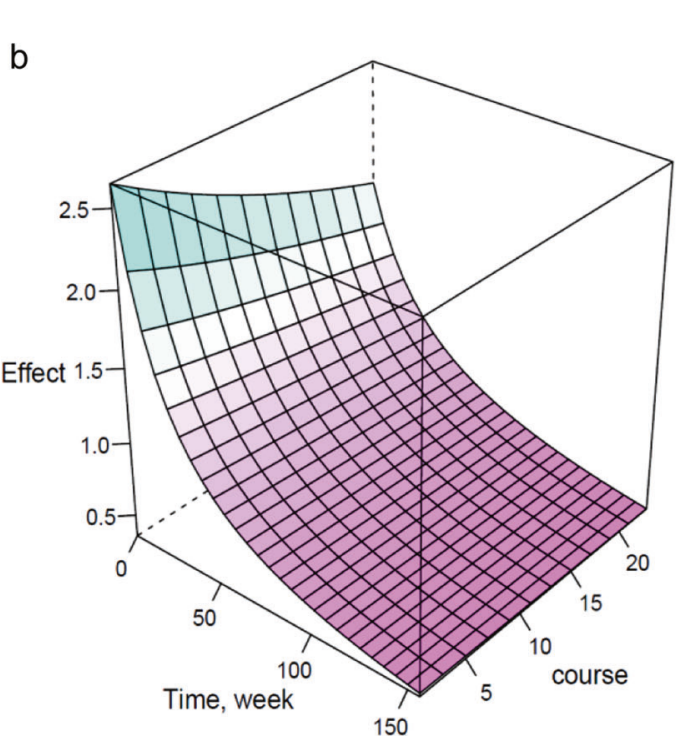

C

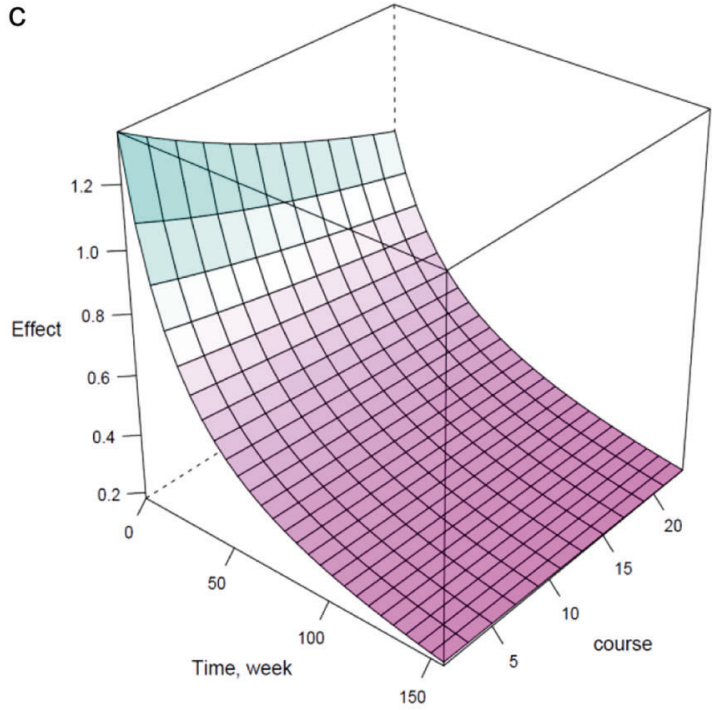

Fig. 4 Predicted three-dimensional model. a oneset frequency-time-effect model of olanzapine; $\mathbf{b}$ course-time-effect model of risperidone in first-episode patient; c: course-time-effect model of risperidone in relapsing patient

\begin{tabular}{|c|c|c|c|}
\hline Time (week) & Olanzapine ( $n$ \%) & Risperidone ( $n$ \%) & Aripiprazole ( $n$ \%) \\
\hline 13 & $5(3.4)$ & $12(7.1)$ & $5(6.8)$ \\
\hline 26 & $44(29.7)$ & 77 (45.8) & $26(35.1)$ \\
\hline 52 & $93(62.8)$ & $128(76.2)$ & $50(67.6)$ \\
\hline 78 & $118(79.2)$ & 144 (85.7) & $60(81.1)$ \\
\hline 104 & $132(89.2)$ & $152(90.5)$ & $66(89.2)$ \\
\hline 130 & $136(91.9)$ & $160(95.2)$ & $67(90.5)$ \\
\hline 156 & 137 (92.6) & $161(95.8)$ & $68(91.9)$ \\
\hline
\end{tabular}

fewer adverse events, such as extrapyramidal adverse reactions, hyperprolactinemia, and metabolic disorders.

There were some limitations in our study. First, the open-label, nonrandomized design of this study was likely to have introduced selection bias. Although we used a set of statistical adjustments for demographic and clinical parameters, bias could not be completely eliminated. Second, in another study, a population pharmacokinetic model was developed to assess the magnitude and variability of exposure to clozapine [35]; however, because we did not collect pharmacokinetic data, we could not build a pharmacokinetic-pharmacodynamic model in this study. Third, because most patients in our study discontinued drug treatment at $\sim 104$ weeks, the drug effect trends after 2 years were unclear. Fourth, although we used different methods to verify the stability of the models, external validation in further studies is required.

\section{CONCLUSIONS}

We established pharmacodynamic models of three atypical antipsychotics based on real-world data. Among the three drugs studied, olanzapine showed the highest efficacy, whereas aripiprazole showed the lowest efficacy. Our current study provided quantitative information on the efficacy of three longterm atypical antipsychotics with regard to the social functioning of real-world schizophrenic patients. 

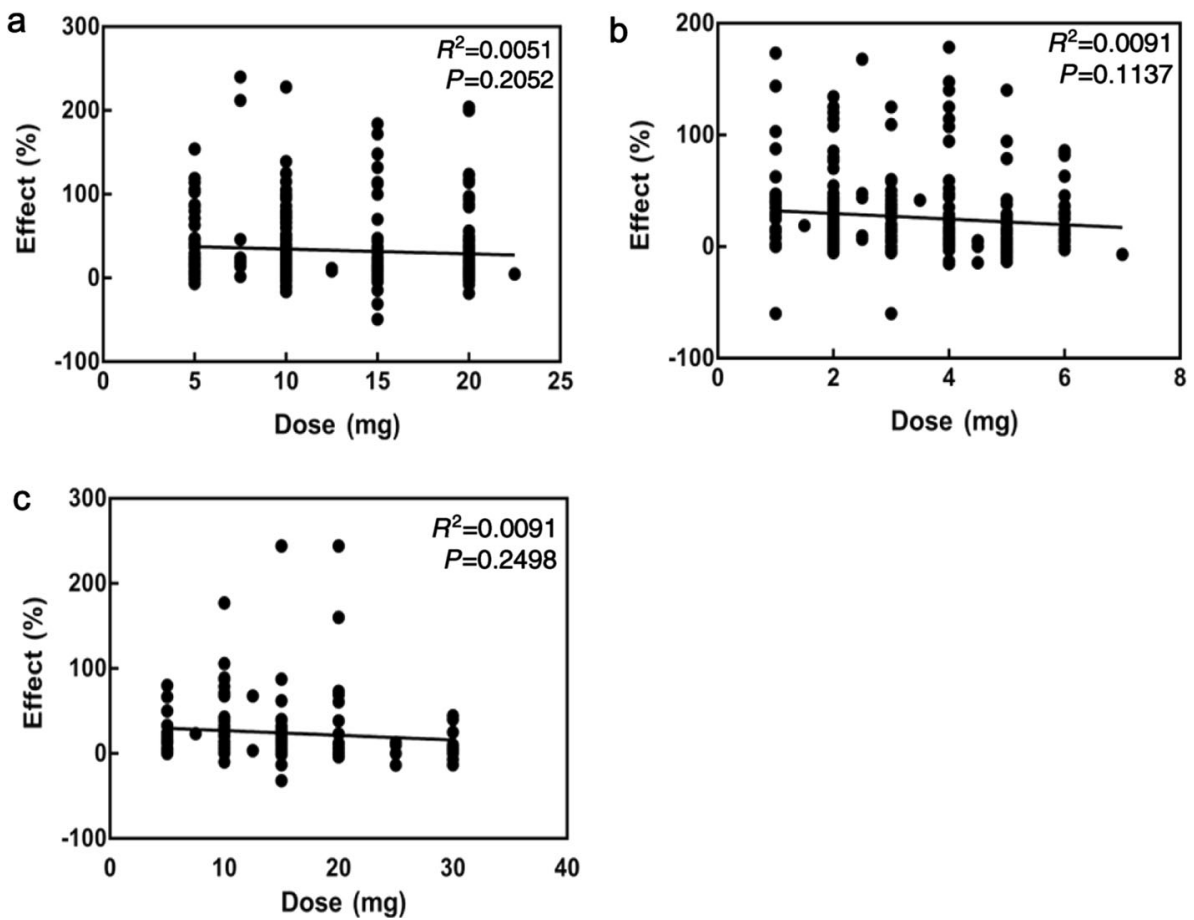

Fig. 5 The relationship between the dose and the predicted $E_{\max }$ value. a olanzapine; $\mathbf{b}$ risperidone; $\mathbf{c}$ aripiprazole

\section{ACKNOWLEDGEMENTS}

This study was supported by the Collaborative Innovation Center for Translational Medicine at the Shanghai Jiao Tong University School of Medicine (TM201506, TM201624), the National Major Project for IND (2018ZX09734-005), and the Clinical Research of Shanghai Jiao Tong University School of Medicine (DLY201620).

\section{AUTHOR CONTRIBUTIONS}

LZ contributed to designing the work, analyzing data, and writing the paper. YGL, YZ and $\mathrm{SH}$ provided approval for publication of the content and searched for the relevant papers. YMY, YL, HW and YQ performed the research. YFS participated in the conception and design of the work. HFL contributed to the design of the study and critically revised the paper for important intellectual content.

\section{ADDITIONAL INFORMATION}

The online version of this article (https://doi.org/10.1038/s41401-019-0285-x) contains supplementary material, which is available to authorized users.

Competing interests: The authors declare no competing interests.

\section{REFERENCES}

1. Leucht S, Corves C, Arbter D, Engel RR, Li C, Davis JM. Second-generation versus first-generation antipsychotic drugs for schizophrenia: a meta-analysis. Lancet. 2009;373:31-41.

2. Lieberman JA, Stroup TS, Mcevoy JP, Swartz MS, Rosenheck RA, Perkins DO, et al. Effectiveness of antipsychotic drugs in patients with chronic schizophrenia. $\mathrm{N}$ Engl J Med. 2005;353:1209-23.

3. Swartz MS, Perkins DO, Stroup TS, Mcevoy JP, Nieri JM, Haak DC. Assessing clinical and functional outcomes in the Clinical Antipsychotic Trials of Intervention Effectiveness (CATIE) schizophrenia trial. Schizophr Bull. 2003;29:33-43.

4. Mcevoy JP, Lieberman JA, Stroup TS, Davis SM, Meltzer HY, Rosenheck RA, et al. Effectiveness of clozapine versus olanzapine, quetiapine, and risperidone in patients with chronic schizophrenia who did not respond to prior atypical antipsychotic treatment. Am J Psychiatry. 2006;163:600-10.

5. Kahn RS, Fleischhacker WW, Boter H, Davidson M, Vergouwe $Y$, Keet IP, et al. Effectiveness of antipsychotic drugs in first-episode schizophrenia and schizophreniform disorder: an open randomised clinical trial. Lancet. 2008;371:1085-97.
6. Burns T, Patrick D. Social functioning as an outcome measure in schizophrenia studies. Acta Psychiatr Scand. 2007;116:403-18.

7. Andreasen NC, Carpenter WT, Kane JM, Lasser RA, Marder SR, Weinberger DR. Remission in schizophrenia: proposed criteria and rationale for consensus. Am J Psychiatry. 2005;162:441-9.

8. Kahn RS. On the continued benefit of antipsychotics after the first episode of schizophrenia. Am J Psychiatry. 2018;175:712-3.

9. Murray RM, Quattrone D, Natesan S, Van Os J, Nordentoft M, Howes O, et al. Should psychiatrists be more cautious about the long-term prophylactic use of antipsychotics? Br J Psychiatry. 2016;209:361-5.

10. Lieberman JA. Effectiveness of antipsychotic drugs in patients with chronic schizophrenia: Efficacy, safety and cost outcomes of CATIE and other trials. J Clin Psychiatry. 2007;68:e04.

11. Stroup TS, Lieberman JA, Mcevoy JP, Swartz MS, Davis SM, Capuano GA, et al Effectiveness of olanzapine, quetiapine, and risperidone in patients with chronic schizophrenia after discontinuing perphenazine: a CATIE study. Am J Psychiatry. 2007; 164:415-27.

12. Galletly C, Castle D, Dark F, Humberstone V, Jablensky A, Killackey E, et al. Royal Australian and New Zealand College of Psychiatrists clinical practice guidelines for the management of schizophrenia and related disorders. Aust $\mathrm{N} \mathrm{Z} \mathrm{J}$ Psychiatry. 2016;50:410-72.

13. Barnes TR. Evidence-based guidelines for the pharmacological treatment of schizophrenia: Recommendations from the British association for psychopharmacology. J Psychopharmacol. 2011;25:567-620.

14. Kay SR, Fiszbein A, Opler LA. The positive and negative syndrome scale (PPANSS) for schizophrenia. Schizophr Bull. 1987;13:261-76.

15. Juckel G, Schaub D, Fuchs N, Naumann U, Uhl I, Witthaus $H$, et al. Validation of the personal and social performance (psp) scale in a German sample of acutely ill patients with schizophrenia. Schizophr Res. 2008;104:287-93.

16. Morosini PL, Magliano L, Brambilla L, Ugolini S, Pioli R. Development, reliability and acceptability of a new version of the dsm-iv social and occupational functioning assessment scale. (SOFAS) to assess routine social functioning. Acta Psychiatr Scand. 2000;101:323-9.

17. Si TM, Shu L, Su YA, Tian CH, Yan J, Cheng J, et al. The Chinese version of the personal and social performance scale (psp): Validity and reliability. Psychiatry Res. 2011;185:275-9.

18. Schwinghammer TL, Kroboth PD. Basic concepts in pharmaco-dynamic modeling. J Clin Pharmacol. 1988;28:388-94.

19. Wählby U, Jonsson EN, Karlsson MO. Comparison of stepwise covariate model building strategies in population pharmacokinetic-pharmacodynamic analysis. AAPS PharmSci. 2002;4:E27. 
20. Parke J, Holford NH, Charles BG. A procedure for generating bootstrap samples for the validation of nonlinear mixed-effects population models. Comput Methods Prog Biomed. 1999;59:19-29.

21. Boter H, Peuskens J, Libiger J, Fleischhacker WW, Davidson M, Galderisi S, et al. Effectiveness of antipsychotics in first-episode schizophrenia and schizophreniform disorder on response and remission: An open randomized clinical trial (EUFEST). Schizophr Res. 2009;115:97-103.

22. Yuan H, Ali MS, Brouwer ES, Girman CJ, Guo JJ, Lund JL, et al. Real-world evidence: What it is and what it can tell us according to the international society for pharmacoepidemiology (ISPE) comparative effectiveness research (CER) special interest group (SIG). Clin Pharmacol Ther. 2018;104:239-41.

23. Vanasse A, Blais L, Courteau J, Cohen AA, Roberge $P$, Larouche A, et al. Comparative effectiveness and safety of antipsychotic drugs in schizophrenia treatment: a real-world observational study. Acta Psychiatr Scand. 2016;134:374-84.

24. Leucht S, Cipriani A, Spineli L, Mavridis D, Orey D, Richter F, et al. Comparative efficacy and tolerability of 15 antipsychotic drugs in schizophrenia: a multipletreatments meta-analysis. Lancet. 2013;382:951-62.

25. Robertson BR, Prestia D, Twamley EW, Patterson TL, Bowie CR, Harvey PD. Social competence versus negative symptoms as predictors of real world social functioning in schizophrenia. Schizophr Res. 2014;160:136-41.

26. Barnes TR, Leeson VC, Mutsatsa SH, Watt HC, Hutton SB, Joyce EM. Duration of untreated psychosis and social function: 1-year follow-up study of first-episode schizophrenia. Br J Psychiatry. 2008;193:203-9.

27. Corcoran CM, Kimhy D, Parrilla-Escobar MA, Cressman VL, Stanford AD, Thompson J, et al. The relationship of social function to depressive and negative symptoms in individuals at clinical high risk for psychosis. Psychol Med. 2011;41:251-61.
28. Robinson DG, Woerner MG, Mcmeniman M, Mendelowitz A, Bilder RM. Symptomatic and functional recovery from a first episode of schizophrenia or schizoaffective disorder. Am J Psychiatry. 2004;161:473-9.

29. Emsley R, Nuamah I, Gopal S, Hough D, Fleischhacker WW. Relapse after antipsychotic discontinuation in schizophrenia as a withdrawal phenomenon vs illness recurrence: a post hoc analysis of a randomized placebo-controlled study. J Clin Psychiatry. 2018;79:17m11874.

30. Andreasen NC, Liu D, Ziebell S, Vora A, Ho BC. Relapse duration, treatment intensity, and brain tissue loss in schizophrenia: a prospective longitudinal MRI study. Am J Psychiatry. 2013;170:609-15.

31. Craig TK, Garety P, Power P, Rahaman N, Colbert S, Fornells-Ambrojo M, et al. The lambeth early onset (LEO) team: randomised controlled trial of the effectiveness of specialised care for early psychosis. BMJ. 2004;329:1067.

32. Takeuchi $H$, Suzuki T, Uchida H, Watanabe K, Mimura M. Antipsychotic treatment for schizophrenia in the maintenance phase: a systematic review of the guidelines and algorithms. Schizophr Res. 2012;134:219-25.

33. Davis SM, Koch GG, Davis CE, Lavange LM. Statistical approaches to effectiveness measurement and outcome-driven re-randomizations in the clinical antipsychotic trials of intervention effectiveness (CATIE) studies. Schizophr Bull. 2003;29:73-80.

34. Yokoi F, Gründer G, Biziere K, Stephane M, Dogan AS, Dannals RF, et al. Dopamine D2 and D3 receptor occupancy in normal humans treated with the antipsychotic drug aripiprazole (OPC 14597): a study using positron emission tomography and $\left[{ }^{11} \mathrm{C}\right]$ raclopride. Neuropsychopharmacology. 2002;27:248-59.

35. Li LJ, Shang DW, Li WB, Guo W, Wang XP, Ren YP, et al. Population pharmacokinetics of clozapine and its primary metabolite norclozapine in Chinese patients with schizophrenia. Acta Pharmacol Sin. 2012;33:1409-16. 